\title{
Field Theoretic Description of Ultrarelativistic Electron-Positron Plasmas
}

\begin{abstract}
Ultrarelativistic electron-positron plasmas can be produced in high-intensity laser fields and play a role in various astrophysical situations. Their properties can be calculated using QED at finite temperature. Here we will use perturbative QED at finite temperature for calculating various important properties, such as the equation of state, dispersion relations of collective plasma modes of photons and electrons, Debye screening, damping rates, mean free paths, collision times, transport coefficients, and particle production rates, of ultrarelativistic electron-positron plasmas. In particular, we will focus on electron-positron plasmas produced with ultra-strong lasers.
\end{abstract}

PACS numbers:

\section{Contents}

\section{Introduction}

II. Equation of state

\section{Collective Phenomena}

IV. Transport properties

\section{Particle production rates}

\section{Non-equilibrium and Finite Chemical Potential}

VII. Conclusions

\section{INTRODUCTION}

Ultrarelativistic plasmas, in which the thermal energy of the particles is much larger than their rest mass energy, were discussed first in the fifties in the context of astrophysics. They occur in the early Universe as well as stellar and galactic high-energy processes (Zel'dovich and Novikov, 1971; Zel'dovich and Novikov, 1983; Raffelt, 1996). For example, ultrarelativistic electron-positron plasmas (EEP) can be created either by high temperatures in supernovae explosions (see e.g. Hardy and Thoma (2001)) or strong magnetic fields in so-called magnetars (Beskin et al., 1993). The theoretical description of ultrarelativistic plasmas is based on transport theory (Silin, 1960) or thermal field theory (Tsytovich, 1961). The latter is an extension of quantum field theory, such as QED or QCD, in vacuum to finite temperature (or chemical potential) describing high-energy particle interactions in matter. For this purpose, two different approaches were developed, the imaginary time formalism (Matsubara, 1955; Kapusta, 1989) and the real time formalism (Landsmann and van Weert, 1987).

In the eighties a new type of ultrarelativistic plasmas became of interest, the quark-gluon plasma (QGP) (Müller, 1985). It corresponds to a new state of matter of deconfined quarks and gluons at extremely high temperatures above $k_{B} T=150 \mathrm{MeV}$. The early Universe should have been in this state for the first few microseconds after the Big Bang. Furthermore, the QGP is expected to be created in relativistic heavy-ion collisions, in particular within the accelerator experiment RHIC (Gyulassy and McLerran, 2005). There a hot and dense fireball ("little bang") of the size of an atomic nucleus is produced, which could be in the QGP phase for less than $10^{-22} \mathrm{~s}$. Therefore the QGP in those experiments cannot be observed directly but its discovery relies on the comparison of theoretically predicted signatures with experimental data ("circumstantial evidence"). For understanding the properties of the QGP and for calculating signatures for its formation, QCD at finite temperature has been applied. Besides a non-perturbative method based on a numerical solution of the QCD equations (lattice QCD), perturbation theory at finite temperature has been used widely (Thoma, 1995a; Le Bellac, 1996). Lattice QCD allows only the calculation of static quantities, and hence not of most of the proposed signatures following from particle production. Perturbative QCD, on the other hand, has been used for computing static as well as dynamic quantities. However, its predictions are limited by the 
fact that the QGP is a strongly-coupled plasma at temperatures achievable in the experiments. (At extremely high temperatures far above the transition temperature perturbative QCD should be reliable due to asymptotic freedom of QCD.) Nonetheless, a wide variety of properties and quantities of the QGP have been considered in this way. It started with the calculation of self-energies and dispersion relations in the high-temperature limit (Klimov, 1982; Weldon, 1982a; Weldon, 1982b). In the high-temperature limit the results differ from the QED results only by trivial factors containing the QCD degrees of freedom (color, flavor). In addition, it can be shown that the gluon self-energy or polarization tensor, which is directly related to the dielectric function (see e.g. Elze and Heinz (1989)), can be derived also within the transport (Vlasov) approach (Silin, 1960), since the high-temperature limit corresponds to the classical limit. Other quantities, however, such as damping or production rates and transport coefficients, require a quantum field theoretic treatment. In particular, a resummation of certain diagrams, the hard thermal loops (HTL), is needed for a consistent description, i.e. to obtain gauge invariant, infrared finite (or improved) results complete to leading order in the coupling constant (Braaten and Pisarski, 1990). Details about quantum field theoretic methods and their application to the physics of the QGP can be found in the review article by Thoma (1995a).

Recently a third possibility to study ultrarelativistic plasmas has been suggested: in extremely strong laser fields the creation of an ultrarelativistic EPP with temperatures around $10 \mathrm{MeV}$ could be realized soon (Liang et al., 1998). For example two opposite, circular polarized laser pulses with a duration of $330 \mathrm{fs}$ and an intensity of $7 \times 10^{21}$ $\mathrm{W} / \mathrm{cm}^{2}$ could hit a thin gold foil. In this way the target electrons can be heated up to about $10 \mathrm{MeV}$ producing an ultrarelativistic EPP by pair creation. The positron density could be about $5 \times 10^{22} \mathrm{~cm}^{-3}$ (Shen and Meyer-ter-Vehn, 2001). This will allow for the first time to study an ultrarelativistic EPP in the laboratory. Therefore predictions of the physical properties of such a system are needed.

The interaction of relativistic electrons and positrons is described by QED. In the presence of a thermal plasma background (heat bath) QED at finite temperature has to be considered. As discussed above, perturbative methods based on the imaginary (Matsubara) or real time formalism have been developed and applied to the physics of a QGP. Here we will transfer the methods and results for properties of a QGP to the case of an EPP, where a perturbative treatment is more reliable than in the case of a strongly coupled QGP. Some applications of thermal field theory to astrophysical plasmas have been discussed before (see e.g. Thoma (2002) and references therein and Altherr and Kraemmer (1992)).

An important difference to non-relativistic ion-electron plasmas (Lifshitz and Pitaevskii, 1981) are the relevant scales. In the non-relativistic case case they are given by the masses of the plasma particles and the temperature $T$. For example, the electron plasma frequency reads

$$
\omega_{p l}=\sqrt{\frac{4 \pi e^{2} \rho_{e}}{m_{e}}}
$$

and the Debye screening length due to the electrons in the plasma

$$
\lambda_{D}=\sqrt{\frac{k_{B} T_{e}}{4 \pi e^{2} \rho_{e}}},
$$

where $\rho_{e}$ is the electron number density, $T_{e}$ the temperature of the electron component, and $m_{e}$ the electron mass.

In an ultrarelativistic plasma with $T \gg m$ the masses can be neglected and the important scales are the temperature $T$, called the hard scale, and the soft scale $e T$, which determines the collective phenomena as we will see below. Here we use natural units, i.e. $\hbar=c=k_{B}=1$, as usual in quantum field theory. In these units $e=0.3$ corresponding to a fine structure constant $\alpha=e^{2} /(4 \pi)=1 / 137$. For converting to SI-units we use $1 \mathrm{MeV} \hat{=} 1.60 \times 10^{-13} \mathrm{~J} \hat{=} 5.08 \times$ $10^{12} \mathrm{~m}^{-1} \hat{=} 1.52 \times 10^{21} \mathrm{~s}^{-1}$.

In the next section we will discuss the equation of state of an equilibrated ultrarelativistic EPP. Then collective phenomena will be considered. Afterwards transport properties and particle production will be discussed. Finally we will describe properties of an EPP which is not in chemical equilibrium as in the case of laser induced plasmas. We will not consider here the formation process and equilibration of an EPP.

Many results presented here can be found in the literature for the case of a QGP (Thoma, 1995a) differing only by numerical factors, e.g. number of degrees of freedom. The purpose of this colloquium is to summarize these results and to extend them to laser induced EPPs as a reference for future experiments. As an example we consider a temperature of $10 \mathrm{MeV}$ as it can be typically realized in laser produced and supernovae EPPs.

Laser produced QED plasmas have also been discussed recently in two review articles (Mourou et al., 2006; Marklund and Shukla, 2006) with emphasis on the production mechanism and non-linear effects. Here, however, we want to focus on the properties of an equilibrated EPP as they can be calculated from perturbative QED. Such an EPP in thermal and chemical equlibrium might be the outcome of future laser experiments if the intensity can be increased further on. As an example, we have chosen the predictions of Shen and Meyer-ter-Vehn (2001) based on a numerical simulation 
(PIC) and cross sections for electron-positron productions, in which two opposite laser beams are focussed on a thin gold foil leading to a chemically non-equiliibrated plasma (see section VI). However, this interesting proposal still waits for its experimental confirmation. Other production mechanisms, based on the Schwinger pair production effect in strong fields (Schwinger, 1951), have shown that pair production can be efficient already far below the Schwinger critical field strength, requiring laser intensities of $5 \times 10^{29} \mathrm{~W} / \mathrm{cm}^{2}$, in the case of time dependent and inhomogeneous fields, e.g. two oppositely directed pulsed laser beams in vacuum (see e.g. Avetissian et al., 2002; Narozhny et al., 2004; Di Piazza, 2004; Dunne and Schubert, 2005; Gies and Klingmuller, 2005; Blaschke et al. 2006a, Blaschke et al., 2006b, Schützhold et al., 2008). Furthermore the pair production in an X-ray free electron laser (XFEL) has been discussed (Ringwald, 2001; Alkofer et al., 2001; Roberts et al., 2002). QED plasmas in strong magnetic fields have been considered by Danielsson and Grasso (1995) and more general in strong electromagnetic fields by Morozov et al. (2002). QED plasmas have also been studied in great detail in the book by Melrose (2008).

\section{EQUATION OF STATE}

In this section we will consider the equation of state of an EPP under the following assumptions:

1. ultrarelativistic EPP, i.e. $T \gg m$,

2. thermal and chemical equilibrium,

3. equal electron and positron density, i.e. vanishing chemical potential,

4. ideal gas, i.e. no interactions in plasma,

5. infinitely extended, homogeneous, and isotropic EPP.

We will relax some of these assumptions in the following sections. According to these assumptions the distribution function of the electrons and positrons is given by the Fermi-Dirac distribution

$$
n_{F}(p)=\frac{1}{e^{p / T}+1}
$$

and of the photons by the Bose-Einstein distribution

$$
n_{F}(p)=\frac{1}{e^{p / T}-1},
$$

where the momentum $p$ is identical to the energy $E$ of the particles in the ultrarelativistic case. It should be noted that the photons are in equilibrium with electrons and positrons under the above assumptions, i.e. the system is actually an electron-positron-photon gas.

The particle and energy density can be calculated by integrating over the distribution functions. In the case of a QGP the results can be found in Müller (1985). The particle number density of the electrons and positrons follows from integrating over the Fermi-Dirac distribution as

$$
\rho_{e}^{e q}=g_{F} \int \frac{d^{3} p}{(2 \pi)^{3}} n_{F}(p)=\frac{3}{\pi^{2}} \zeta(3) T^{3}=0.37 T^{3}
$$

where $g_{F}=4$ is the number of degrees of freedom corresponding to the electrons and positrons in the two spin states. Assuming a temperature of $T=10 \mathrm{MeV}$, we find $\rho_{e}^{e q}=370 \mathrm{MeV}^{3}=4.9 \times 10^{40} \mathrm{~m}^{-3}$.

The photon density follows accordingly by integrating over the Bose-Einstein distribution with $g_{B}=2$ degrees of freedom corresponding to the two polarization states as $\rho_{p h}^{e q}=\left(2 / \pi^{2}\right) \zeta(3) T^{3}=0.24 T^{3}$.

The energy density of the electron-positron-photon gas is obtained from

$$
\epsilon^{e q}=g_{F} \int \frac{d^{3} p}{(2 \pi)^{3}} p n_{F}(p)+g_{B} \int \frac{d^{3} p}{(2 \pi)^{3}} p n_{B}(p)=\frac{11 \pi^{2}}{60} T^{4}=1.81 T^{4}
$$

where the photons contribute $36 \%$ to the energy density. Here the Boltzmann law, $\epsilon^{e q} \sim T^{4}$, holds also for the fermions because we neglected their masses.

For $T=10 \mathrm{MeV}$ we find $\epsilon^{e q}=3.8 \times 10^{29} \mathrm{~J} \mathrm{~m}^{-3}$. In a volume of $10^{12} \mathrm{~m}^{3}$ (corresponding to the size of a neutron star) the total thermal energy of the EPP is $3.8 \times 10^{41} \mathrm{~J}$, which corresponds to about $10 \%$ of the entire energy (without neutrinos) released in a supernova type II explosion. In a volume of $1 \mu \mathrm{m}^{3}$ there is still an energy of $3.8 \times 10^{11} \mathrm{~J}$ contained.

The Coulomb coupling parameter of the EPP, which is a measure for the non-ideal behavior of a plasma (Ichimaru, 1982), is given by $\Lambda=e^{2} /(d T)$, where $d \simeq \rho_{e}^{e q-1 / 3}=2.7 \times 10^{-14} \mathrm{~m}$ is the interparticle distance. For $T=10 \mathrm{MeV}$ we find $\Lambda=5.3 \times 10^{-3}$ which shows that the EPP is a weakly coupled plasma in contrast to the QGP where $\Lambda=O(1)$ 


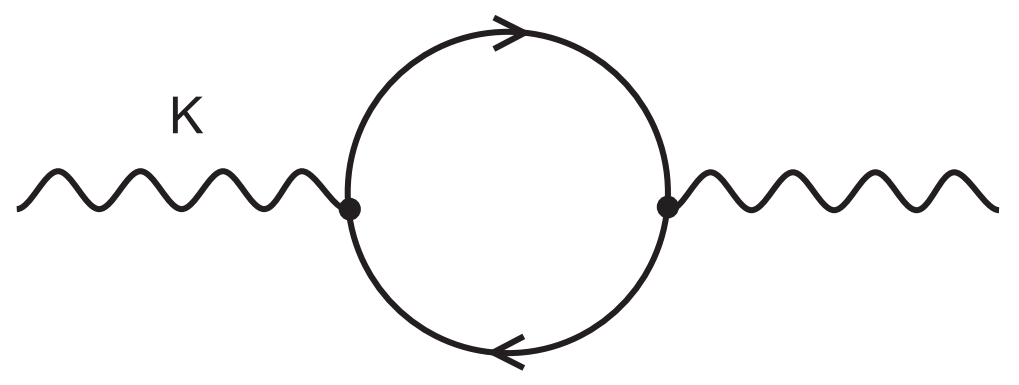

FIG. 1: One-loop polarization tensor.

(Thoma, 2005). Therefore the ideal gas results for the equation of state derived above are a good approximation. After all, interactions in the EPP play an important role, for example, for the collective behavior of the plasma as discussed in the next section and for equilibration of the plasma. Obviously, the interaction can be treated by perturbation theory.

\section{COLLECTIVE PHENOMENA}

Interactions between particles can be separated into two classes: individual collisions between the particles and long-range interactions of particles with the medium (Lifshitz and Pitaevskii, 1981). The latter ones lead to collective effects, which are characteristic for plasmas. The crucial quantity from which the collective phenomena are derived is the dielectric tensor relating the macroscopic electric field $D_{i}$ in the medium to the external field $E_{i}(i=x, y, z)$, i.e. in momentum space

$$
D_{i}(\omega, \mathbf{k})=\sum_{j} \epsilon_{i j}(\omega, \mathbf{k}) E_{j}(\omega, \mathbf{k})
$$

In the case of an isotropic medium it depends only on $k=|\mathbf{k}|$ and has two independent components

$$
\epsilon_{i j}(\omega, k)=\epsilon_{T}(\omega, k)\left(\delta_{i j}-\frac{k_{i} k_{j}}{k^{2}}\right)+\epsilon_{L}(\omega, k) \frac{k_{i} k_{j}}{k^{2}}
$$

The dielectric tensor is closely related to the polarization tensor or photon self-energy by (see e.g. Elze and Heinz $(1989))$

$$
\begin{aligned}
& \epsilon_{L}(\omega, k)=1-\frac{\Pi_{L}(\omega, k)}{k^{2}} \\
& \epsilon_{T}(\omega, k)=1-\frac{\Pi_{T}(\omega, k)}{\omega^{2}},
\end{aligned}
$$

where $\Pi_{L}$ and $\Pi_{T}$ are the longitudinal and transverse components of the polarization tensor, respectively.

The lowest order diagram for the polarization tensor is shown in Fig.1. Assuming the external momentum to be soft, i.e. $\omega$ and $k$ to be much smaller than $T$ and the internal loop momenta to be hard (HTL approximation) an analytic result can be found using the real or imaginary time formalism (Klimov, 1982; Weldon, 1982a)

$$
\begin{aligned}
& \Pi_{L}(\omega, k)=-3 m_{p h}^{2}\left(1-\frac{\omega}{2 k} \ln \frac{\omega+k}{\omega-k}\right), \\
& \Pi_{T}(\omega, k)=\frac{3}{2} m_{p h}^{2} \frac{\omega^{2}}{k^{2}}\left[1-\left(1-\frac{k^{2}}{\omega^{2}}\right) \frac{\omega}{2 k} \ln \frac{\omega+k}{\omega-k}\right],
\end{aligned}
$$

where $m_{p h}=e T / 3$ is called the effective photon mass. For $T=10 \mathrm{MeV}$ we get $m_{p h}=1 \mathrm{MeV}$.

The hard thermal loop approximation, which is identical to the high-temperature limit, produces consistently the lowest order result in finite temperature QED (Braaten and Pisarski, 1990). The dielectric functions following from (9) and (10) can also be derived from the classical Vlasov equation together with the Maxwell equations (Silin, 1960), since the high-temperature limit corresponds to the classical limit. 


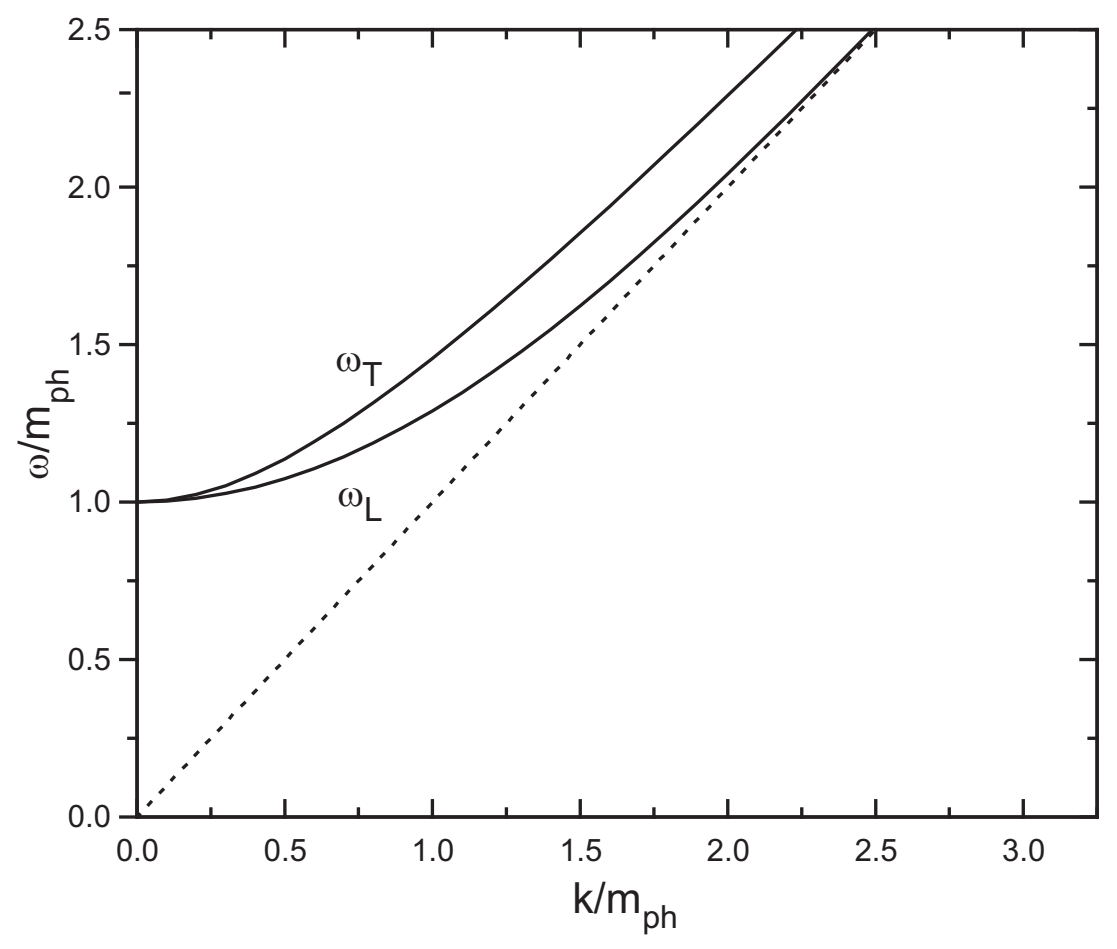

FIG. 2: Photon dispersion relation.

The dispersion relations of collective plasma modes, i.e. propagation of electromagnetic waves in the plasma, can be found by using the Maxwell equation, leading to (see e.g. Carrington et al. (2004))

$$
\begin{aligned}
\epsilon_{L}(\omega, k) & =0, \\
\epsilon_{T}(\omega, k) & =\frac{k^{2}}{\omega^{2}} .
\end{aligned}
$$

Combining (9), (10), and (11) gives the dispersion relations $\omega_{L, T}(k)$ of the transverse as well as longitudinal plasma waves as shown in Fig.2. The longitudinal branch, which does not exist in vacuum, is called plasmon as in the case of non-relativistic plasmas. The transverse branch does not play a role in non-relativistic plasmas but is equally important as the longitudinal one in relativistic plasmas. Both branches start at the plasma frequency $\omega_{p l}=\omega_{L, T}(k=0)=m_{p h}$ (Kajantie and Kapusta, 1985). Consequently the collective plasma waves have soft momenta of the order $e T$. At high momenta $k \gg m_{p h}$ the transverse mode approaches the free dispersion $\omega_{T}=k$, corresponding to a real photon in vacuum, whereas the longitudinal mode disappears, i.e. its spectral strength is exponentially suppressed (Pisarski, 1989). For $T=10 \mathrm{MeV}$ we find $\omega_{p l}=1.5 \times 10^{21} \mathrm{~s}^{-1}$.

Electromagnetic plasma waves in an ultrarelativistic EPP have also been discussed by Medvedev (1999). For the plasma frequency the same dependence on the temperature and coupling constant was found (see eq.(3) in Medvedev (1999)) if one uses the equilibrium number density for the photons proportional to $T^{3}$ (see above) there. However, the numerical pre-factor there is wrong and only one branch of plasma waves was discussed.

Another important quantity which can be derived from the polarization or dielectric tensor is the Debye screening length, entering the Yukawa potential of a heavy, non-relativistic test charge in the EPP. The Debye screening length is given by the static limit of the longitudinal component of the polarization tensor $1 / \Pi_{L}(\omega=0)$ (Kajantie and Kapusta, 1985), leading to $\lambda_{D}=1 /\left(\sqrt{3} m_{p h}\right)$, which is $1.1 \times 10^{-13} \mathrm{~m}$ for $T=10 \mathrm{MeV}$.

Finally from (10) we see that the polarization tensor and the dielectric function become imaginary for $\omega^{2}<k^{2}$, i.e. below the light cone $\omega=k$, corresponding to Landau damping (Pisarski, 1988). We also observe that the plasma waves calculated at lowest order perturbation theory are undamped since they are located at $\omega>k$. This changes, however, at higher orders where the dispersion relation can intersect the light cone (Carrington et al., 2004). It is interesting to note that all these results are apart from some numerical color and flavor factors also valid for collective gluon modes in the QGP in the high-temperature limit (Thoma, 1995a).

A complete new phenomenon that does not appear in non-relativistic plasmas is the existence of fermionic plasma waves because all fermion masses are much too large in the non-relativistic case. Their dispersion relations follow 
from the pole of the electron propagator containing the electron self-energy. Using again the hard thermal loop approximation the electron self-energy reads $\left(P=\left(p_{0}, \mathbf{p}\right), p=|\mathbf{p}|\right)$ (Klimov, 1982; Weldon, 1982b)

$$
\Sigma(P)=-a\left(p_{0}, p\right) P^{\mu} \gamma_{\mu}-b\left(p_{0}, p\right) \gamma_{0}
$$

with

$$
\begin{aligned}
& a\left(p_{0}, p\right)=\frac{1}{4 p^{2}}\left[\operatorname{tr}\left(P^{\mu} \gamma_{\mu} \Sigma\right)-p_{0} \operatorname{tr}\left(\gamma_{0} \Sigma\right)\right], \\
& b\left(p_{0}, p\right)=\frac{1}{4 p^{2}}\left[P^{2} \operatorname{tr}\left(\gamma_{0} \Sigma\right)-p_{0} \operatorname{tr}\left(P^{\mu} \gamma_{\mu} \Sigma\right)\right],
\end{aligned}
$$

where the traces over the $\gamma$ matrices are given by

$$
\begin{aligned}
\operatorname{tr}\left(P^{\mu} \gamma_{\mu} \Sigma\right) & =4 m_{F}^{2} \\
\operatorname{tr}\left(\gamma_{0} \Sigma\right) & =2 m_{F}^{2} \frac{1}{p} \ln \frac{p_{0}+p}{p_{0}-p}
\end{aligned}
$$

with the effective electron mass $m_{F}=e T / \sqrt{8}$ (Klimov, 1982; Weldon, 1982b), which is $1.1 \mathrm{MeV}$ at $T=10 \mathrm{MeV}$.

The full electron propagator in the helicity representation is given by (Braaten et al., 1990)

$$
S^{\star}(P)=\frac{1}{2 D_{+}(P)}\left(\gamma_{0}-\hat{p} \cdot \gamma\right)+\frac{1}{2 D_{-}(P)}\left(\gamma_{0}+\hat{p} \cdot \gamma\right),
$$

where

$$
D_{ \pm}(P)=-p_{0} \pm p+\frac{1}{4 p}\left[ \pm \operatorname{tr}\left(P^{\mu} \gamma_{\mu} \Sigma\right)-\left( \pm p_{0}-p\right) \operatorname{tr}\left(\gamma_{0} \Sigma\right)\right]
$$

Again these results agree with the ones for quarks in the QGP apart from numerical pre-factors.

The dispersion relations following from the pole of this propagator are shown in Fig.3. Again two branches show up, one with a positive ratio of the helicity to chirality $\left(\omega_{+}\right)$following from $D_{+}=0$, the other one with a negative ratio $\left(\omega_{-}\right)$following from $D_{-}=0$, called plasmino (Braaten et al., 1990). The plasmino branch $\omega_{-}$, which does not exist in vacuum, shows an interesting behavior, namely a minimum at $k=0.41 m_{F}$. The same behavior of the quark dispersion in the QGP has been found. In this case sharp peaks in the dilepton production rate can appear due to the minimum in the plasmino branch, which leads to van Hove singularities (Braaten et al., 1990; Peshier and Thoma, 2000). Whether something similar could be observed in the EPP, e.g. in the electron spectrum, is a very interesting question which should be investigated in detail. It could open the exciting possibility to observe a new collective plasma wave, the plasmino, experimentally in a laser induced EPP.

The collective quantities derived here perturbatively from the dielectric functions are linear phenomena. Nonlinear collective effects in electron-positron plasmas, have been considered in a number of publications, e.g. soliton formation (Lontano et al., 2001), nonlinear Alfvén waves (Zhao et al., 1994), nonlinear photon interactions (Tajima and Taniuti, 1990), plasma enhanced photon splitting (Brodin et al., 2007), and nonlinear self-modulation of radio pulses (Chian and Kennel, 1983).

\section{TRANSPORT PROPERTIES}

Now we want to consider the interaction and properties of particles in the plasma with hard momenta, i.e. of the order of $T$ or larger. In particular we are interested in damping and transport rates, mean free paths, collision times, energy losses of these particles and other transport properties such as the shear viscosity of the EPP. All these quantities have been calculated perturbatively in high-temperature QCD (Thoma, 1995a). As in the case of the collective phenomena, the results can be almost directly taken from the QCD calculations.

It was shown by Braaten and Pisarski (1990) that a consistent treatment of gauge theories such as QED at finite temperature, i.e. for obtaining results that are gauge independent, infrared finite, and complete to leading order, require the use of an effective perturbation theory using resummed Green functions based on the HTL approximation (HTL resummation technique). The HTL method relies on the assumption that one can distinguish between soft and hard momenta, i.e. $e T \ll T$. This is approximately fulfilled for QED, where $e=0.3$, but not in QCD, where the corresponding coupling constant $g>1$.

The damping rate of an electron or positron in the EPP is defined as the imaginary part of the dispersion relation $\omega_{L, T}(p)$. To lowest order it follows from the elastic scattering diagram of Fig.4. In the case of a hard electron or 


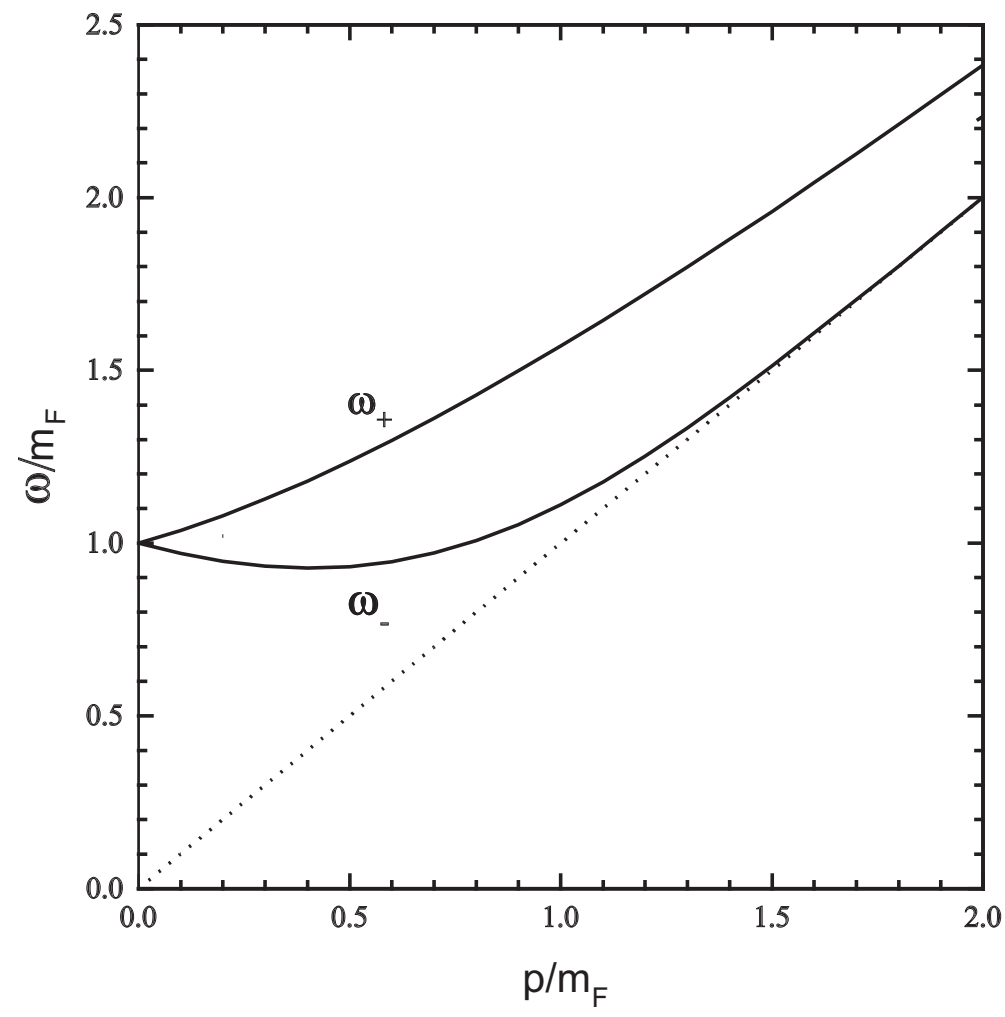

FIG. 3: Electron dispersion relation.

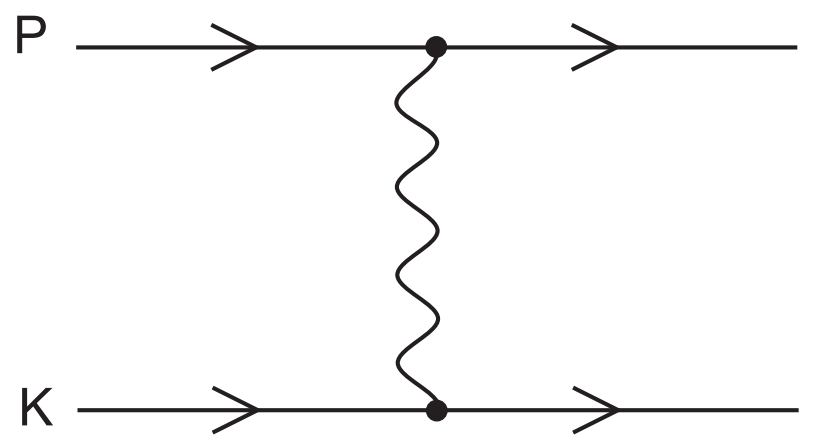

FIG. 4: Lowest order diagram for electron-electron scattering.

positron with momenta of the order of $T$ or higher it exhibits a quadratic infrared (IR) divergence which can be reduced to a logarithmic one using a HTL resummed photon propagator. This logarithmic singularity is expected to be cut-off by higher order contributions leading to (Thoma, 1995a)

$$
\gamma_{e}=\frac{e^{2} T}{4 \pi} \ln \frac{1}{e}
$$

within logarithmic accuracy, i.e. the constant under the logarithm is not determined. For $T=10 \mathrm{MeV}$ we obtain $\gamma_{e}=86 \mathrm{keV}$, which is much smaller than $\omega_{p l}=1 \mathrm{MeV}$, showing that the EPP is not overdamped.

Physically more important are the transport rates $\Gamma$ which are related to the mean free path and collision time of electrons and positrons in the EPP. They differ from the damping rate in cutting off the long range interactions with small scattering angles $\theta$ by a factor $(1-\cos \theta)$ under the integral defining the rate (Lifshitz and Pitaevskii, 1981). This leads to an improvement of the IR behavior (logarithmic instead of quadratic singularity in perturbation theory) and a finite result using the HTL method. Logarithmic divergent quantities can be treated consistently by splitting them into a soft part and a hard part, where the soft part is calculated using the HTL resummation technique (Braaten and Yuan, 1991). For the transport rate we find to logarithmic accuracy by extending the QCD results in 

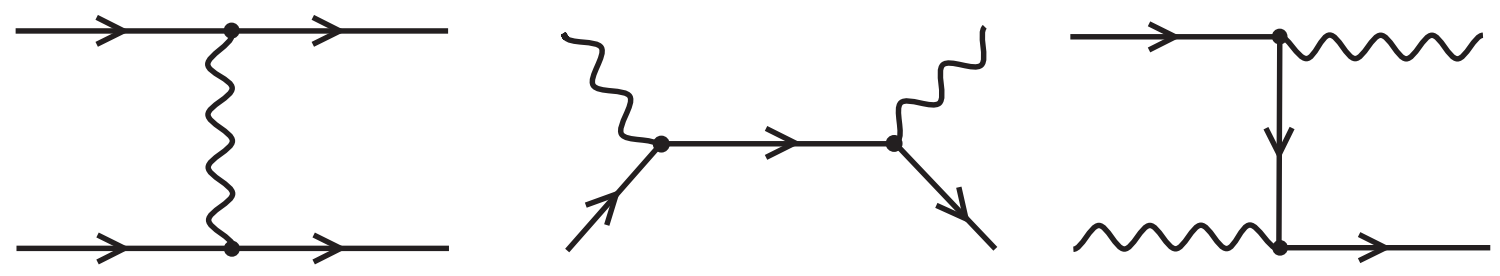

FIG. 5: Diagrams defining the collisional energy loss.

Thoma (1994) to QED

$$
\Gamma_{e}=\frac{e^{4} T^{3}}{3 \pi s} \ln \frac{1}{e}
$$

where the Mandelstam variable $s=(P+K)^{2}$ is the square of the sum of the four momenta of the incoming particles in the scattering diagram of Fig.4. For deriving the above result we replaced in (15) of Thoma (1994) $C_{A} g^{2}$ by $e^{2}$ and $m_{g}$ by $m_{p h}$. For going beyond the logarithmic approximation, which is valid within about a factor of two, one has to calculate explicitly the hard contribution from the QED scattering diagrams as it was done by Thoma (1994) for the QCD case. For thermal particles we replace $s$ by its thermal average $\langle s\rangle=2\langle p\rangle_{e}\langle k\rangle_{e} \simeq 19.3 T^{2}$ (Thoma, 1994), where $\langle p\rangle_{e}=\langle k\rangle_{e}=\epsilon_{e}^{e q} / \rho_{e}^{e q}=3.11 T$. Assuming again $T=10 \mathrm{MeV}$, we get $\Gamma_{e}=0.54 \mathrm{keV}$.

The mean free path $\lambda_{e}^{m f p}$ and collision time $\tau_{e}$ of the plasma particles (electrons and positrons) are given by the inverse of the transport rate $1 / \Gamma_{e}$, leading to $\lambda_{e}^{m f p}=0.37 \mathrm{~nm}$ and $\tau_{e}=1.2 \times 10^{-18} \mathrm{~s}$ at $T=10 \mathrm{MeV}$.

In a non-relativistic plasma the shear viscosity can be estimated from elementary kinetic theory as (Reif, 1965)

$$
\eta_{i}=\frac{1}{3} \sum_{i} \rho_{i}\left\langle p_{i}\right\rangle \lambda_{i}^{m f p}
$$

where the sum is performed over the various components of the system. In an relativistic plasma the coefficient $1 / 3$ should be replaced by 4/15 (de Groot et al., 1980). Using the mean free path following from (18), the density of (5), and the thermal momentum $\langle p\rangle_{e}=3.11 T$, the shear viscosity is given by (within logarithmic accuracy)

$$
\eta_{e}=\frac{55.8 T^{3}}{e^{4} \ln (1 / e)}
$$

At $T=10 \mathrm{MeV}$ the shear viscosity coefficient is $\eta_{e}=7.9 \times 10^{10} \mathrm{~Pa} \mathrm{~s}$.

Another quantity of interest in a plasma is its stopping power or the energy loss of an energetic particle in the plasma. There are two contributions, namely the energy loss by collisions and the radiative one by bremsstrahlung. In a relativistic plasma the latter one becomes important. The relevance of these contributions in the QGP has been discussed controversially (Mustafa and Thoma, 2005). The collisional energy loss is given by the mean energy transfer divided by the mean free path leading to (Braaten and Thoma, 1991)

$$
\frac{d E}{d x}=\frac{1}{v} \int d \gamma \omega
$$

where $v$ is the particle velocity, $\gamma$ the damping or interaction rate proportional to the plasma density and the collision cross section, and $\omega$ the energy transfer from the energetic particle to plasma particle in the collision. Using for the collision cross section the lowest order diagrams in Fig.5, the collisional energy of a muon with mass $M$ in an EPP has been calculated by Braaten and Thoma (1991) applying the HTL resummation technique

$$
\frac{d E}{d x}=\frac{e^{4} T^{2}}{24 \pi}\left(\frac{1}{v}-\frac{1-v^{2}}{2 v^{2}} \ln \frac{1+v}{1-v}\right)\left(\ln \frac{E}{M}+\ln \frac{1}{e}+A(v)\right)
$$

where $A(v)$ is a slowly varying function of the muon velocity $v$ between 1.3 and 1.5 .

The collisional energy loss of an electron with energy $E \gg T$ is approximately given by (Braaten and Thoma, 1991)

$$
\frac{d E}{d x}=\frac{e^{4} T^{2}}{48 \pi} \ln \frac{15.3 E}{e^{2} T} .
$$

In Braaten and Thoma (1991) the factor 15.3 was replaced by 7.6 due to an numerical error which was corrected in Thoma (1995a). This leads to an energy loss of $200 \mathrm{MeV} / \mathrm{nm}$ for an electron or positron with an energy of $E=100$ 

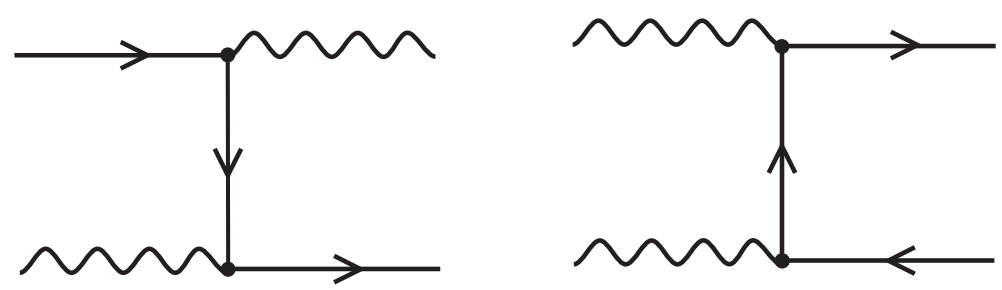

FIG. 6: Diagrams defining the photon damping rate.

$\mathrm{MeV}$ at $T=10 \mathrm{MeV}$, showing that such an electron is stopped (thermalized) within a fraction of a nanometer. (Note that the plasma density, proportional to $T^{3}$, on which the energy loss depends via the mean free path is hidden in the temperature dependence of the energy loss.) Recently it has been shown that this calculation can be improved by taking into account additional diagrams changing this result slightly (Peigne and Peshier, 2008). So far no calculations of the radiative energy loss in an EPP have been performed to our knowledge.

The damping rate of a photon in an EPP follows from the diagram in Fig.6, where a HTL resummed electron propagator has to be used in case of soft momenta of the exchanged electron (positron). In contrast to the electron damping rate, the photon rate is infrared finite using the HTL method due to the presence of an electron propagator in Fig.6 instead of the photon propagator in Fig.4. Hence there is no need to cut off the long range interaction introducing a transport cross section. The result for a photon with energy $E=p$ reads (Thoma, 1995b)

$$
\Gamma_{p h}=\frac{e^{4} T^{2}}{64 \pi E} \ln \frac{3.88 E}{e^{2} T} .
$$

The mean free path and the collision time of photons in an EPP are given by $1 / \Gamma_{p h}$. For a thermal photon with the mean momentum $\langle p\rangle_{p h}=\epsilon_{p h}^{e q} / \rho_{p h}^{e q}=2.75 T$ at $T=10 \mathrm{MeV}$ the mean free path $\lambda_{p h}^{m f p}=0.28 \mathrm{~nm}$ and the collision time $\tau_{p h}=9.4 \times 10^{-19} \mathrm{~s}$ follow. Actually the damping rate given in (24) is a lower limit as higher order effects will enlarge it. As a matter of fact, the photon production rate in a QGP, which is the inverse process of the damping rate (Thoma, 1995b), was shown to be about a factor of 2 larger taking bremsstrahlung into account (Arnold et al., 2001).

For the viscosity of the photon component using the above mean free path, the photon density (see above), and the mean photon energy $\langle p\rangle_{p h}=2.75 T$ we find

$$
\eta_{p h}=\frac{48.7 T^{3}}{e^{4} \ln (3.27 / e)}
$$

corresponding to $3.5 \times 10^{10} \mathrm{~Pa}$ s at $T=10 \mathrm{MeV}$. Hence the viscosity of the EPP $\eta=\eta_{e}+\eta_{p h}$ has similar contributions from the electrons and photons.

A more advanced calculation of the total viscosity of the EPP based on the Kubo formula yields within logarithmic accuracy (Arnold et al., 2000)

$$
\eta=\frac{188 T^{3}}{e^{4} \ln (1 / e)}
$$

This result is about a factor of 1.5 larger than the one presented here based on the elementary kinetic theory, which is typically valid within a factor of 2 (Reif, 1965).

\section{PARTICLE PRODUCTION RATES}

At high temperatures above $10 \mathrm{MeV}$ also other particle species will be produced, e.g. muons with a mass of $m_{\mu}=106$ $\mathrm{MeV}$. Their rate follows to lowest order from the diagram in Fig.7 (Born term). We assume that $m_{e} \ll T \ll m_{\mu}$ holds. The first inequality implies that the electron mass can be put to zero and the latter inequality implies that muons are not equilibrated. Then the muon production rate to lowest order $\left(e^{-} e^{+} \rightarrow \gamma^{*} \rightarrow \mu^{-} \mu^{+}\right)$is given by

$$
\frac{d N}{d^{4} x d^{4} p}=\frac{\alpha^{2}}{24 \pi^{4}}\left(1+\frac{2 m_{\mu}^{2}}{M^{2}}\right)\left(1-\frac{4 m_{\mu}^{2}}{M^{2}}\right)^{1 / 2} \frac{T}{p} \frac{1}{\exp (E / T)-1} \ln \frac{1+\exp [-(E+p) /(2 T)]}{1+\exp [-(E-p) /(2 T)]}
$$




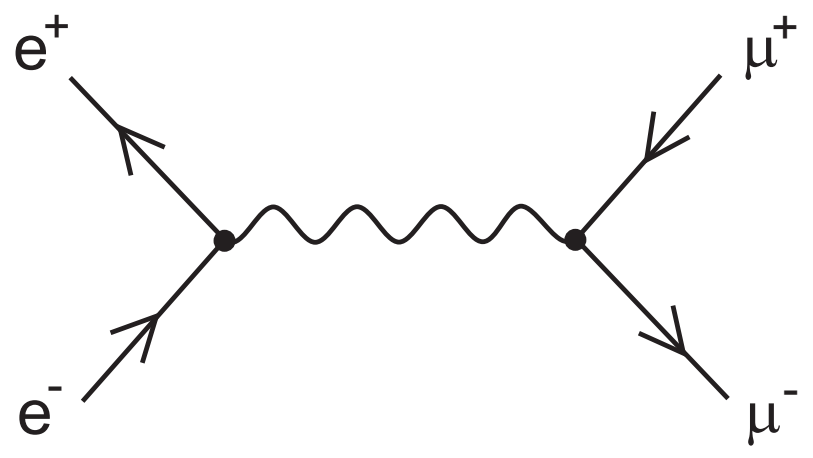

FIG. 7: Lowest order contribution of the muon production.

where $M^{2}=E^{2}-p^{2}$ is the invariant mass of the virtual photon $\gamma^{*}, E$ its energy and $p=|\mathbf{p}|$ its momentum. This formula was derived by combining the cross section for the process in Berends et al. (1973) with the production rate in Cleymans et al. (1987) where the quark-antiquark annihilation process $q \bar{q} \rightarrow \mu^{-} \mu^{+}$was considered. Here we assumed the chemical potential $\mu=0$. The only difference to the process considered here comes from the fractional charge of the quarks, i.e. we use here $\sum_{i} e_{i}^{2}=1$, and from the number of colors in the distribution functions in $(6 a, b)$ of Cleymans et al. (1987), i.e. dividing the QGP rate by a factor of 9 . In addition a factor 3/2 appears in the electron-positron annihilation cross section - see (6) of Berends et al. (1973) after integrating over the angle compared to the quark-antiquark annihilation cross section in (5) of Cleymans et al. (1987), using the relative velocity of the ultrarelativistic particles $v_{q \bar{q}}=1$. Because of $M^{2}=E^{2}-p^{2}>4 m_{\mu}^{2}$ the rate is suppressed exponentially for temperatures below $2 m_{\mu}$.

In order to obtain the spectrum from this formula one has to integrate over the space-time volume, taking into account the space-time evolution by using, for example, a hydrodynamical model. The total muon yield then follows from integrating the spectrum over the energy and momentum of the virtual photon.

At temperatures above $10 \mathrm{MeV}$ also hadron production becomes important, in particular pion production (Kuznetsova et al., 2008).

\section{NON-EQUILIBRIUM AND FINITE CHEMICAL POTENTIAL}

EPPs produced in strong laser fields are probably not in complete equilibrium. For example, it has been predicted by Shen and Meyer-ter-Vehn (2001) that an positron density of about $5 \times 10^{28} \mathrm{~m}^{-3}$ at a temperature of $10 \mathrm{MeV}$ can be reached. This density deviates from the equilibrium density (5) by 12 orders of magnitude. In the following we will therefore assume that the EPP produced by lasers is in thermal but not in chemical equilibrium. Then we can replace the distribution functions for the electrons by Fermi-Dirac distributions multiplied by a fugacity factor $\lambda$ describing the deviation from chemical equilibrium, $f_{F}(p)=\lambda n_{F}(p)$. This assumption has been used for example for describing the chemical equilibration of the QGP in ultrarelativistic heavy-ion collisions (Biró et al., 1993). The fugacity is given by the ratio of the experimental to equilibrium particle density, since the experimental density follows from integrating over the non-equilibrium distribution, i.e.

$$
\rho_{\text {exp }}=g_{F} \int \frac{d^{3} p}{(2 \pi)^{3}} \lambda n_{F}(p)=\lambda \rho_{e q} \Rightarrow \lambda=10^{-12}
$$

Using the real time formalism, QED perturbation theory and the HTL method can also be extended to nonequilibrium situations like the one discussed above (Carrington et al., 1999). For example, the effective photon mass is given now by

$$
m_{p h}^{2}=\frac{4 e^{2}}{3 \pi^{2}} \int_{0}^{\infty} d p p f_{F}(p)
$$

For $T=10 \mathrm{MeV}$ we then find for the non-equilibrium photon mass $m_{p h}^{n o n e q}=\sqrt{\lambda} m_{p h}=1 \mathrm{eV}$ and the plasma frequency $\omega_{p l}^{n o n e q}=1.5 \times 10^{15} \mathrm{~Hz}$. The Debye screening length in such an EPP is $\lambda_{D}=0.1 \mu \mathrm{m}$. In order to speak of a plasma with collective behavior its dimension $L$ should be much larger than $\lambda_{D}$, i.e. at least of the order of $1 \mu \mathrm{m}$.

Furthermore, an anisotropic EPP can also be described by quantum field theoretic methods (Mrówczyński and Thoma, 2000). In this case instabilities can occur (Mrówczyński and Thoma, 2007). 
Finally a possible difference between the positron density and the electron density can be treated by introducing a finite chemical potential $\mu$, i.e. using the distribution

$$
n_{F}(p)=\frac{1}{e^{(p \pm \mu) / T}+1}
$$

for the electrons (negative sign) and positrons (positive sign). Such a difference comes from the fact that the laser produced EPP is embeded in a hot electron and cold ion background of the target. Therefore there will be an excess of electrons over positrons in the hot, relativistic EPP. The methods described above, such as the HTL resummation, can be generalized easily to this case (Vija and Thoma, 1995). For example, the energy density is given by

$$
\epsilon^{e q}=\frac{11 \pi^{2}}{60} T^{4}+\frac{1}{2} T^{2} \mu^{2}+\frac{1}{4 \pi^{2}} \mu^{4}
$$

or the effective photon energy by

$$
m_{p h}^{2}=\frac{e^{2} T^{2}}{9}\left(1+\frac{3 \mu^{2}}{\pi^{2} T^{2}}\right) .
$$

The muon production rate for finite chemical potential can be found by replacing the last three factors in (27) by (13) of Cleymans et al. (1987).

\section{CONCLUSIONS}

The properties of ultrarelativistic EPPs can be treated by QED perturbation theory at finite temperature similarly as the QGP by QCD using the imaginary or real time formalism. A consistent treatment requires the use of the HTL resummation technique for many quantities under consideration. Many results can be more or less directly taken from the corresponding QGP calculation. The examples discussed here are the equation of state, the dielectric tensor, the dispersion relations of photons and electrons, the Debye screening length, damping and transport rates, mean free paths, collision times, shear viscosity, stopping power, and the muon production rate. In particular collective phenomena, such as plasma waves, are of interest. In an EPP completely new phenomena appear, namely collective fermion modes associated with the possibility to observe van Hove singularities.

EPPs produced by strong lasers open the unique possibility to investigate these plasmas and their properties which also exist in astrophysical systems, e.g. supernova explosions. However, current predictions indicate that laser induced EPPs are not in chemical equilibrium. Their properties therefore require an extension of perturbative QED and the HTL resummation technique to non-equilibrium situations, which is possible within the real time formalism. As a first application we discussed the Debye screening length in such an EPP, demonstrating that the EPP should have a spatial extension of at least one micron to exhibit a characteristic plasma behavior.

Here we have considered a number of relevant properties of an ultrarelativistic, equilibrated EPP as a reference for future laboratory experiments and astrophysical EPPs and gave numbers for a plasma temperature of $10 \mathrm{MeV}$. The results are summarized in the table below.

New results, which have not been published so far, are the electron transport rate (18), the electron viscosity (22), and the photon viscosity (27). Also new is the application of the results, derived here or compiled from the literature, to relativistic laser plasmas as presented in the third row of the table and in the discussion of non-equilibrium effects.

Further quantities, e.g. the radiative energy loss, or higher order corrections, e.g. beyond the leading logarithm approximation, to the quantities considered above could be evaluated in a similar way as discussed above. Also an extension of the properties discussed in sections II - V to the case of a chemical non-equilibrated EPP would be of interest. Finally we did not consider here the formation of an EPP in a strong laser field, e.g. by the Schwinger mechanism, and its equilibration.

Acknowledgment: I would like to thank D. Habs, J. Rafelski, I. Kouznetsova, and G. Moore for helpful discussions and hints. 
Table: Properties of an EPP

\begin{tabular}{|c|c|c|}
\hline Quantity & Formula & Value at $T=10 \mathrm{MeV}$ \\
\hline Electron-Positron Density & $\rho_{e}^{e q}=3 / \pi^{2} \zeta(3) T^{3}$ & $4.9 \times 10^{40} \mathrm{~m}^{-3}$ \\
\hline Photon Density & $\rho_{p h}^{e q}=2 / \pi^{2} \zeta(3) T^{3}$ & $3.2 \times 10^{40} \mathrm{~m}^{-3}$ \\
\hline Electron-Positron Energy Density & $\epsilon_{e}^{e q}=7 \pi^{2} / 60 T^{4}$ & $2.4 \times 10^{29} \mathrm{~J} \mathrm{~m}^{-3}$ \\
\hline Photon Energy Density & $\epsilon_{p h}^{e q}=\pi^{2} / 15 T^{4}$ & $1.4 \times 10^{29} \mathrm{~J} \mathrm{~m}^{-3}$ \\
\hline Total Energy Density & $\epsilon^{e q}=11 \pi^{2} / 60 T^{4}$ & $3.8 \times 10^{29} \mathrm{~J} \mathrm{~m}^{-3}$ \\
\hline Thermal electron momentum & $\langle p\rangle_{e}=\epsilon_{e}^{e q} / \rho_{e}^{e q}=3.11 T$ & $31 \mathrm{MeV}$ \\
\hline Thermal photon momentum & $\langle p\rangle_{p h}=\epsilon_{p h}^{e q} / \rho_{p h}^{e q}=2.75 T$ & $28 \mathrm{MeV}$ \\
\hline Interparticle distance & $d \simeq \rho_{e}^{e q-1 / 3}$ & $2.7 \times 10^{-14} \mathrm{~m}$ \\
\hline Coulomb Coupling Parameter & $\Lambda=e^{2} /(d T)$ & $5.3 \times 10^{-3}$ \\
\hline Effective Photon Mass & $m_{p h}=e T / 3$ & $1 \mathrm{MeV}$ \\
\hline Plasma Frequency & $\omega_{p l}=m_{p h}$ & $1.5 \times 10^{21} \mathrm{~s}^{-1}$ \\
\hline Debye Screening Length & $\lambda_{D}=1 /\left(\sqrt{3} m_{p h}\right)$ & $1.1 \times 10^{-13} \mathrm{~m}$ \\
\hline Effective Electron Mass & $m_{F}=e T /(2 \sqrt{2})$ & $1.1 \mathrm{MeV}$ \\
\hline Electron Damping Rate & $\gamma_{e}=e^{2} T /(4 \pi) \ln (1 / e)$ & $86 \mathrm{keV}$ \\
\hline Electron Transport Rate & $\Gamma_{e}=e^{4} T^{3} /(3 \pi s) \ln (1 / e)$ & $0.54 \mathrm{keV}$ for $s=19.3 T^{2}$ \\
\hline Photon Damping Rate & $\Gamma_{p h}=e^{4} T^{2} /(64 \pi E) \ln \left(3.88 E / e^{2} T\right)$ & $0.70 \mathrm{keV}$ for $E=2.75 T$ \\
\hline Electron Mean Free Path & $\lambda_{e}^{m f p}=1 / \Gamma_{e}$ & $0.37 \mathrm{~nm}$ \\
\hline Photon Mean Free Path & $\lambda_{p h}^{m f p}=1 / \Gamma_{p h}$ & $0.28 \mathrm{~nm}$ \\
\hline Electron Collision Time & $\tau_{e}^{p n}=1 / \Gamma_{e}$ & $1.2 \times 10^{-18} \mathrm{~s}$ \\
\hline Photon Collision Time & $\tau_{p h}=1 / \Gamma_{p h}$ & $9.4 \times 10^{-19} \mathrm{~s}$ \\
\hline Electron Viscosity & $\eta_{e}=55.8 T^{3} /\left[e^{4} \ln (1 / e)\right]$ & $7.9 \times 10^{10} \mathrm{~Pa} \mathrm{~s}$ \\
\hline Photon Viscosity & $\eta_{p h}=48.7 T^{3} /\left[e^{4} \ln (3.27 / e)\right]$ & $3.5 \times 10^{10} \mathrm{~Pa} \mathrm{~s}$ \\
\hline Total Viscosity & $\eta=\eta_{e}+\eta_{p h}$ & $(1.1-1.6) \times 10^{11} \mathrm{~Pa} \mathrm{~s}$ \\
\hline Electron Energy Loss & $d E / d x=e^{4} T^{2} /(48 \pi) \ln \left(15.3 E / e^{2} T\right)$ & $\begin{array}{l}200 \mathrm{MeV} / \mathrm{nm} \text { for } E=100 \\
\mathrm{MeV}\end{array}$ \\
\hline
\end{tabular}

\section{References}

Alkofer, R., M. B. Hecht, C. D. Roberts, S. M. Schmidt, and D. V. Vinnik, 2001, Phys. Rev. Lett. 87, 193902.

Altherr, T., and U. Kraemmer, 1992, Astropart. Phys. 1, 133.

Arnold, P., L. G. Yaffe, and G. D. Moore, 2000, JHEP 0011, 001.

Arnold, P., G. D. Moore, and L. Yaffe, 2001, JHEP 0112, 009.

Avetissian, H. K., A. K. Avetissian, G. F. Mkrtchian, and K. V. Sedrakian, 2002, Phys. Rev. E 66, 016502.

Berends, F. A., K. J. F. Gaemers, and R. Gastmans, 1973, Nucl. Phys. B 57, 381.

Beskin, V. S., A. V. Gurevich, and Y. N. Istomin, 1993, Physics of the Pulsar Magnetosphere, (Cambridge University Press, Cambridge).

Biró, T. S., E. van Doorn, B. Müller, M. H. Thoma, and X. N. Wang, 1993, Phys. Rev. C 48, 1275.

Blaschke, D. B., A.V. Prozorkevich, C. D. Roberts, S. M. Schmidt, and S. A. Smolyansky, 2006a, Phys. Rev. Lett. 96, 140402.

Blaschke, D. B., A.V. Prozorkevich, S. A. Smolyansky, and A. V. Tarakanov, 2006b, J. Phys: Conf. Ser. 35, 121.

Braaten, E., and R. D. Pisarski, 1990, Nucl. Phys. B 337, 569.

Braaten, E., R. D. Pisarski, and T. C. Yuan, 1990, Phys. Rev. Lett. 64, 2242.

Braaten, E., and M. H. Thoma, 1991, Phys. Rev. D 44, 1298.

Braaten, E., and T. C. Yuan, 1991, Phys. Rev. Lett. 66, 2183.

Brodin, G., M. Marklund, B. Eliasson, and P. K. Shukla, 2007, Phys. Rev. Lett. 98, 125001.

Carrington, M. E., H. Defu, and M. H. Thoma, 1999, Eur. Phys. J. C 67, 347.

Carrington, M. E., T. Fugleberg, D. Pickering, and M. H. Thoma, 2004, Can. J. Phys. 82, 671.

Chian, A. C.-L., and C. F. Kennel, 1983, Astrophys. Space Sci. 97, 9.

Cleymans, J., J. Fingberg, and K. Redlich, 1987, Phys. Rev. D 35, 2153.

Danielsson, U. H., and D. Grasso, 1995, Phys. Rev. D 52, 2533.

Di Piazza, A., 2004, Phys. Rev. D 70, 053013.

Dunne, G. V., and C. Schubert, 2005, Phys. Rev D 72, 105004.

Elze, H. T., and U. Heinz, 1989, Phys. Rep. 183, 81.

Gies, H., and K. Klingmuller, 2005, Phys. Rev. D 72, 065001.

de Groot, S. R., W. A. van Leeuwen, and C. G. van Weert, 1980, Relativistic Kinetic Theory (North-Holland, Amsterdam).

Gyulassy, M. and L. McLerran, 2005, Nucl. Phys. A 750, 30. 
Hardy, S. J., and M. H. Thoma, 2001, Phys. Rev. D 63, 025014.

Ichimaru, S., 1982, Rev. Mod. Phys. 54, 1017.

Kajantie, K., and J. I. Kapusta, 1985, Ann. Phys. (N.Y.) 160, 477.

Kapusta, J. I., 1989, Finite Temperature Field Theory (Cambridge University Press, New York).

Klimov, V. V., 1982, Zh. Eksp. Teor. Fiz. 82, 336 [Sov. Phys. JETP 55, 199 (1982)].

Kuznetsova, I., D. Habs, and J Rafelski, 2008, Phys. Rev. D 78, 014027.

Landsmann, N. P., and C. G. van Weert, 1987, Phys. Rep. 145, 141.

Le Bellac, M., 1996, Thermal Field Theory (Cambridge University Press, Cambridge).

Liang, E. P., S. C. Wilks, and M. Tabak, 1998, Phys. Rev. Lett. 81, 4887.

Lifshitz, E. M., and L. P. Pitaevskii, 1981, Physical Kinetics (Pergamon Press, Oxford).

Lontano, M., S. Bulanov, and J. Koga, 2001, Phys. Plasmas 8, 5113.

Marklund, M., and P. K. Shukla, 2006, Rev. Mod. Phys. 78, 591.

Matsubara, T., 1955, Progr. Theor. Phys. 14, 351.

Melrose, D. B., 2008, Quantum Plasmaphysics - Unmagnetized Plasmas (Springer, Berlin).

Medvedev, M. V., 1999, Phys. Rev. E 59, R4766.

Morozov, V. G., G. Röpke, and A. Höll, 2002, Theor. Math. Phys. 131, 812; ibid. 132, 1029.

Mourou, G. A., T. Tajima, and S. V. Bulanov, 206, Rev. Mod. Phys. 78, 309.

Mrówczyński, S., and M. H. Thoma, 2000, Phys. Rev. D 62, 036011.

Mrówczyński, S., and M. H. Thoma, 2007, Annu. Rev. Nucl. Part. Sci. 57, 61.

Mustafa, M. G., and M. H. Thoma, 2005, Acta Phys. Hung. A 22, 93.

Müller, B., 1985, The Physics of the Quark-Gluon Plasma, Lecture Notes in Physics 225 (Springer, Berlin).

Narozhny, N. B., S. S. Bulanov, V. D. Mur, and V. S. Popov, 2004, Phys. Lett. A 330, 1.

Peigne, S. and A. Peshier, 2008, Phys. Rev. D 77, 014015.

Peshier, A., and M. H. Thoma, 2000, Phys. Rev. Lett. 84, 841.

Pisarski, R. D., 1988, Nucl. Phys. B 309, 476.

Pisarski, R. D., 1989, Physica A 158, 146.

Raffelt, G., 1996, Stars as Laboratories for Fundamental Physics, (Univ. Chicago Press, Chicago).

Reif, F., 1965, Fundamentals of Statistical and Thermal Physics, (McGraw-Hill, New York).

Ringwald, A., 2001, Phys. Lett. B 510, 107.

Roberts, C. D., S. M. Schmidt, and D. V. Vinnik, 2002, Phys. Rev. Lett. 89, 153901.

Schützhold, R., Gies H., and G. Dunne, eprint arXiv:0807.0754.

Schwinger, J., 1951, Phys. Rev. 82, 664.

Shen, B., and J. Meyer-ter-Vehn, 2001, Phys. Rev. E 65, 016405.

Silin, V. P., 1960, J. Exptl. Theoret. Phys. (U.S.S.R.) 38, 1577 [Sov. Phys. JETP 11, 1136 (1960)].

Tajima, T. and T. Taniuti, 1990, Phys. Rev. A 42, 3587.

Thoma, M. H., 1994, Phys. Rev. D 49, 451.

Thoma, M. H., 1995a, in Quark-Gluon Plasma 2, edited by R.C. Hwa (World Scientific, Singapore, 1995), p.51 [eprint hep-ph/9503400.

Thoma, M. H. 1995b, Phys. Rev D 51, 862.

Thoma, M. H., 2002, in Space Science Series of ISSI, Vol.14, edited by P. Jetzer, K. Pretzl, and R. von Steiger (Kluwer Academic Publishers, Dordrecht), p.141 [eprint astro-ph/0104078].

Thoma, M. H., 2005, J. Phys. G 31, L7 and Erratum J. Phys. G 31, 539.

Tsytovich, V. N., 1961, J. Exptl. Theoret. Phys. (U.S.S.R.) 40, 1775 [Sov. Phys. JETP 13, 1249 (1961)].

Vija, H., and M. H. Thoma, 1995, Phys. Lett. B 342, 212.

Weldon, H. A., 1982a, Phys. Rev. D 26, 1394.

Weldon, H. A., 1982b, Phys. Rev. D 26, 2789.

Zel'dovich, Y. B., and Novikov, 1971, Relativistic Astrophysics, Vol. 1: Stars and Relativity, (Univ. Chicago Press, Chicago).

Zel'dovich, Y. B., and Novikov, 1983, Relativistic Astrophysics, Vol. 2: the Structure and Evolution of the Universe, (Univ. Chicago Press, Chicago).

Zhao, J., K. I. Nishikawa, J. I. Sakai, and T. Neubert, 1994, Phys. Plasmas 1, 103. 